\title{
EMPREGO DO SAL DI-SÓDICO DE EDTA COMO PADRÃO NO PREPARO DE SOLUÇÕES
}

\author{
Paulo H. Pereira da Silva*, Andrea Oliveira, Lívia Soman de Medeiros e Eduardo F. de Almeida Neves ${ }^{\dagger}$ \\ Departamento de Química, Universidade Federal de São Carlos, CP 676, 13560-970 São Carlos - SP, Brasil
}

Recebido em 15/2/06; aceito em 7/7/06; publicado na web em 19/1/07

\begin{abstract}
USE OF EDTA DISODIUM SALT AS STANDARD FOR PREPARATION OF SOLUTIONS. The hydrated sodium salt of EDTA, $\mathrm{Na}_{2} \mathrm{H}_{2} \mathrm{Y} \cdot 2 \mathrm{H}_{2} \mathrm{O}$, cannot be used as a primary standard for titrations due to uncertainties in the water content. An alkalimetric titration of the homogenized solid in the presence of a small excess of $\mathrm{BaCl}_{2} \cdot 2 \mathrm{H}_{2} \mathrm{O}$ allows one to titrate quantitatively the released two hydrogen cations with end-point indication by phenolphthalein or potentiometry. This leads one to calculate the average molar mass of the reagent and its water content, allowing to use it to prepare EDTA standard solutions. One titrated sample led to the formula $\mathrm{Na}_{2} \mathrm{H}_{2} \mathrm{Y} \cdot 1.876 \mathrm{H}_{2} \mathrm{O}$, and $370.01 \mathrm{~g} \cdot \mathrm{mol}^{-1}$ for the average molar mass.
\end{abstract}

Keywords: EDTA; standard; potentiometry.

\section{INTRODUÇÃO}

Schwarzebbach ${ }^{1}$ foi o pioneiro no campo de desenvolvimento das chamadas complexonas, isto é, agentes complexométricos policoordenativos, capazes de formar com cátions metálicos complexos de alta estabilidade na proporção molar 1:1, prestando-se a titulações complexométricas de grande número de cátions metálicos e, indiretamente, de alguns ânions. Outros pesquisadores que lhe seguiram os passos, como Ringbom ${ }^{2}$ e Martell ${ }^{3,4}$, tiveram papel importante no desenvolvimento deste setor da Química Analítica.

$\mathrm{O}$ ácido etilenodiaminotetraacético, $\mathrm{H}_{4} \mathrm{Y}$, comumente representado pela sigla EDTA, é sem dúvida a complexona mais utilizada e estudada dentro desta classe, apresentando aplicação ampla e geral em análise química $^{1-3}$ por ser um produto industrial de baixo custo, especialmente na forma do sal di-sódico, $\mathrm{Na}_{2} \mathrm{H}_{2} \mathrm{Y} \cdot 2 \mathrm{H}_{2} \mathrm{O}$. Há relatos na literatura ${ }^{5,6}$ de que tanto o ácido de EDTA na forma livre, $\mathrm{H}_{4} \mathrm{Y}$, quanto seu sal di-sódico, $\mathrm{Na}_{2} \mathrm{H}_{2} \mathrm{Y} \cdot 2 \mathrm{H}_{2} \mathrm{O}$, podem ser utilizados como padrões primários, pois estão disponíveis comercialmente em elevado grau de pureza. O sal disódico hidratado, bem solúvel, é a forma indicada, mas as condições para sua obtenção como padrão primário são criticáveis. Investigações realizadas por Durval ${ }^{7}$ via curvas termogravimétricas (TG) têm mostrado que, em relação ao sal di-sódico de EDTA, o ácido em sua forma livre pode ser, a princípio, considerado melhor padrão primário, por não possuir águas de hidratação, não ser higroscópio e quando submetido à secagem suporta temperaturas elevadas, em torno de $130-145^{\circ} \mathrm{C}$. Entretanto, é pouco solúvel em água.

Já o sal di-sódico de EDTA, sob condições normais atmosféricas, ou seja, à temperatura de $25^{\circ} \mathrm{C}$, contém em sua composição cerca de 0,3-0,5\% de umidade, em excesso, além da água de hidratação do sólido ${ }^{8}$. Para preparo da solução de EDTA, o sólido deve ser seco a uma temperatura de $80^{\circ} \mathrm{C}$, por um período de dois a três dias, caso contrário pode perder águas de hidratação. $\mathrm{O}$ sólido quando seco deve ter sua composição concordante com a fórmula $\mathrm{Na}_{2} \mathrm{H}_{2} \mathrm{Y} \cdot 2 \mathrm{H}_{2} \mathrm{O}$ (massa molar de $372,24 \mathrm{~g} \mathrm{~mol}^{-1}$ ). Entretanto, em conceituados textos de Química Analítica ${ }^{7,9}$ não há recomendação deste sal hidratado como padrão primário, diante da instabilidade de sua composição. Portando, recomenda-se sempre a padronização das soluções de EDTA.

*e-mail: paulaoqui@dq.ufscar.br

${ }^{\dagger}$ In memoriam
A completa desidratação do sal di-sódico de EDTA pode ser obtida aquecendo-o a uma temperatura em torno de $130-150{ }^{\circ} \mathrm{C}^{7}$. A composição do sal anidro, obtido nestas condições, concorda exatamente com a fórmula $\mathrm{Na}_{2} \mathrm{H}_{2} \mathrm{Y}$. Entretanto, estudos termogravimétricos ${ }^{7,10}$ confirmaram que o sal di-sódico anidro não deve ser considerado um padrão primário, devido ser extremamente higroscópio. Diante da inviabilidade do uso do EDTA na sua forma di-sódica anidra, $\mathrm{Na}_{2} \mathrm{H}_{2} \mathrm{Y}$, ou mesmo na sua forma ácida, $\mathrm{H}_{4} \mathrm{Y}$, como padrão primário, este trabalho tem como objetivo propor uma metodologia que possibilite a determinação do grau de hidratação do sal di-sódico e, conseqüentemente, a determinação da massa molar média deste reagente. $\mathrm{O}$ conhecimento preciso da massa molar média permite empregar o sólido como um padrão secundário no preparo das soluções de EDTA. Esta metodologia relativamente simples, desenvolvida por este grupo de pesquisa ${ }^{11,12}$, está fundamentada em titulações alcalimétricas das soluções de EDTA na presença de excesso de íons de bário ou de cálcio.

\section{PARTE EXPERIMENTAL}

Todos os reagentes comerciais empregados foram de pureza analítica P.A. Todas as soluções foram preparadas utilizando água destilada, previamente borbulhada com nitrogênio, a fim de se eliminar a presença de $\mathrm{CO}_{2}$ dissolvido.

Como titulante, foi preparada uma solução padrão de $\mathrm{NaOH}$ $0,1896 \mathrm{~mol} \mathrm{~L}^{-1}$ livre de íons de carbonato ${ }^{11,13}$. No preparo desta solução, a remoção de íons de carbonato foi realizada adicionando-se uma suspensão de $\mathrm{Ca}(\mathrm{OH})_{2}$, sendo a massa de $\mathrm{CaO}$ utilizada de 0,5 g, para uma alíquota de $200 \mathrm{~mL}$ de solução de $\mathrm{NaOH}$ em torno de $0,5 \mathrm{~mol} \mathrm{~L}^{-1}$, filtrando-a a seguir. Por simples diluição desta solução livre de íons carbonato, foi preparada uma outra solução mais diluída em nível $0,2 \mathrm{~mol} \mathrm{~L}^{-1}$, a qual foi padronizada pelo processo convencional de uso do padrão primário, hidrogenoftalato de potássio?.

Para determinação do teor de hidratação e também da massa molar média, pesou-se cerca de $30 \mathrm{~g}$ de um sal di-sódico de EDTA, seguindo-se sua homogenização em almofariz. Pesaram-se, em 4 algarismos significativos, massas deste sal di-sódico de EDTA homogeneizado entre 0,70 e $0,71 \mathrm{~g}$. A cada massa pesada adicionou-se cerca de $50 \mathrm{~mL}$ de água destilada, juntando-se uma massa de $\mathrm{BaCl}_{2} \cdot 2 \mathrm{H}_{2} \mathrm{O}$ da ordem de $0,8 \mathrm{~g}$. Titulou-se potenciometricamente o ácido liberado na solução resultante, com solução padrão de 
hidróxido de sódio livre de carbonato, previamente preparada. As titulações foram realizadas em triplicata e as medidas potenciométricas foram obtidas utilizando-se um eletrodo de referência $\mathrm{Ag} /$ $\mathrm{AgCl}, \mathrm{KCl} 3,0 \mathrm{~mol} \mathrm{~L}^{-1}$ combinado da marca Orion. Este eletrodo estava acoplado a um pHmetro da marca Digmed DM 20 de sensibilidade $0,1 \mathrm{mV}$.

A recristalização do sal di-sódico foi realizada obtendo-se primeiramente uma solução saturada a $80^{\circ} \mathrm{C}$. Após seu resfriamento à temperatura ambiente, a adição de igual volume de etanol leva à precipitação imediata do sólido hidratado, completada em refrigerador, por $24 \mathrm{~h}$, à temperatura de $8{ }^{\circ} \mathrm{C}$. Completada esta etapa, separou-se o sólido por filtração, sendo então seco em estufa a 45 ${ }^{\circ} \mathrm{C}$, por um período de 3 dias.

\section{RESULTADOS E DISCUSSÃO}

Sabe-se que em Química Analítica a utilização como padrão primário do reagente EDTA principalmente na sua forma di-sódica, $\mathrm{Na}_{2} \mathrm{H}_{2} \mathrm{Y}$ ou $\mathrm{Na}_{2} \mathrm{H}_{2} \mathrm{Y} \cdot 2 \mathrm{H}_{2} \mathrm{O}$, não é recomendada devido à instabilidade de sua composição na forma anidra, muito higroscópica e, também, por desvios no grau de hidratação, o que afeta a massa molar média deste composto. Sendo assim, soluções deste reagente sempre devem ser padronizadas antes de serem empregadas em qualquer tipo de análise química. Recentemente foi proposto por $\mathrm{Ne}$ ves et al. ${ }^{12}$ um novo procedimento de padronização de soluções de sal di-sódico de EDTA, baseado na titulação alcalimétrica dos íons de hidrogênio liberados pelo EDTA, na presença de excesso de íons de bário ou de cálcio.

Segundo esta metodologia, quando íons de bário (ou cálcio) são adicionados a uma solução de EDTA, $\mathrm{H}_{2} \mathrm{Y}^{2-}$, um complexo de [EDTABário] é formado, na relação estequiométrica molar 1:1, e uma pequena quantidade de íons hidrogênio é, então, liberada, segundo o Equilíbrio 1:

$\mathrm{Ba}^{2+}+\mathrm{H}_{2} \mathrm{Y}^{2-} \leftrightarrows \mathrm{BaY}^{2-}+2 \mathrm{H}^{+} \mathrm{K}=\mathrm{K}_{3} \mathrm{~K}_{4} \beta_{\mathrm{BaY} 2-}=2,2 \times 10^{-9} \mathrm{~mol} \mathrm{~L}^{-1}(1)$

sendo $\mathrm{K}_{3}$ e $\mathrm{K}_{4}$ as constantes de ionização do $\mathrm{H}_{2} \mathrm{Y}^{2-}$ e $\beta_{\mathrm{BaY2}-}$ a constante de formação do complexo do $\mathrm{Ba}^{2+}$ com $\mathrm{Y}^{4}$.

Na titulação da solução de EDTA, com solução padrão de hidróxido de sódio, íons de hidrogênio presentes na solução começam a ser neutralizados e o equilíbrio acima é deslocado para a direita. Segundo Neves et al. ${ }^{12}$, neste caso, uma nova constante $\mathrm{K}_{\text {total }}$ é definida, de alta magnitude, resultante da combinação do Equilíbrio 1 com o produto iônico da água, $1 / \mathrm{K}_{\mathrm{w}}{ }^{2}$ :

$\begin{array}{lr}\mathrm{Ba}^{2+}+\mathrm{H}_{2} \mathrm{Y}^{2-} \leftrightarrows \mathrm{BaY}^{2-}+2 \mathrm{H}^{+} & \mathrm{K}=2,2 \times 10^{-9} \mathrm{~mol} \mathrm{~L}^{-1} \\ 2 \mathrm{H}^{+}+2 \mathrm{OH}^{-} \leftrightarrows 2 \mathrm{H}_{2} \mathrm{O} & 1 / \mathrm{K}_{\mathrm{w}}{ }^{2}=1,0 \times 10^{28} \mathrm{~mol}^{-4} \mathrm{~L}^{4} \\ \mathrm{Ba}^{2+}+\mathrm{H}_{2} \mathrm{Y}^{2-}+2 \mathrm{OH}^{-} \leftrightarrows \mathrm{BaY}^{2-}+2 \mathrm{H}_{2} \mathrm{O} \mathrm{K}_{\text {total }}=2,2 \mathrm{x}^{19} \mathrm{~mol}^{-3} \mathrm{~L}^{3}(2)\end{array}$

O alto valor da constante global assim calculada, 2,2x10 ${ }^{19} \mathrm{~mol}^{-3}$ $\mathrm{L}^{3}$, indica a viabilidade da titulação alcalimétrica de soluções de EDTA na sua forma di-sódica na presença de um algum excesso de íons de bário, onde o ponto de equivalência pode ser monitorado empregando-se um indicador ácido-base, como a fenolftaleína (faixa de $\mathrm{pH}$ de 8,3-10) ou por método potenciométrico utilizando, neste caso, um eletrodo de vidro. A quantidade adicionada de $\mathrm{BaCl}_{2} \cdot 2 \mathrm{H}_{2} \mathrm{O}$ não é crítica, entretanto recomenda-se que a proporção molar $\left[\mathrm{Ba}^{2+}\right] /\left[\mathrm{H}_{2} \mathrm{Y}^{2-}\right]$ deva ser no mínimo $1: 1$, estendendo-se até relação molar $3: 1^{12}$.

Contudo, há possibilidade da titulação direta do EDTA di-sódico hidratado na sua forma sólida, ao invés da solução deste reagente. Desta forma, aplicando-se alguns cálculos relativamente simples é possível determinar satisfatoriamente o grau de hidratação do sal di-sódico do EDTA, como também sua massa molar média, o que o caracterizaria como um sólido padrão. Temos particular interesse no emprego do EDTA como sólido assim padronizado, pelo fato de que estamos trabalhando com soluções deste reagente parcialmente neutralizadas, nas formas de $\mathrm{H}_{2} \mathrm{Y}^{2-} / \mathrm{HY}^{3-}$ com $50 \%$ de neutralização do íon $\mathrm{H}_{2} \mathrm{Y}^{2-}$ e $\mathrm{HY}^{3-} / \mathrm{Y}^{4-}$, com $75 \%$ de neutralização, constituindo sistemas auto tamponantes. Desta maneira, para preparo das soluções parcialmente neutralizadas, pesa-se o sólido com base na sua massa molar média previamente determinada, adiciona-se o volume desejado de hidróxido de sódio padrão, livre de carbonato, para um determinado volume fixo.

Na padronização do sólido homogeneizado, conforme descrito anteriormente na seção experimental, uma massa de EDTA é pesada, dissolvida em um pequeno volume de água destilada e, posteriormente, titulada com solução de hidróxido de sódio. Esta titulação pode ser realizada de forma visual ou pelo método potenciométrico (Figura 1).
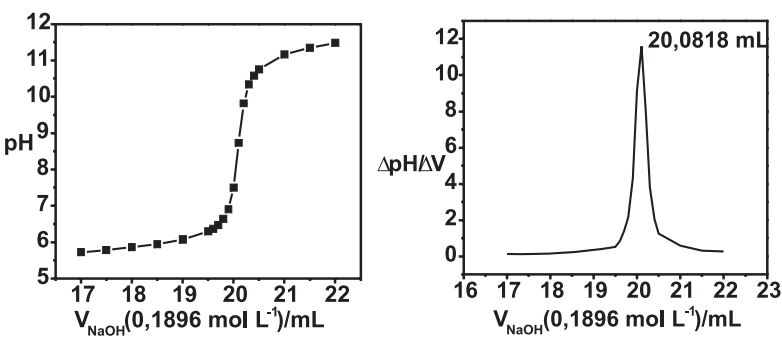

Figura 1. Curvas de uma titulação potenciométrica de 0,7044 g de EDTA di-

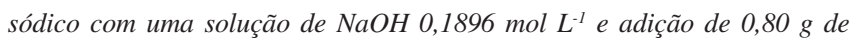
$\mathrm{BaCl}_{2} \cdot 2 \mathrm{H}_{2} \mathrm{O}$

Na titulação de certa massa do sal di-sódico, a proporção molar existente é de 2 mols de $\mathrm{OH}^{-}$por mol de $\mathrm{H}_{2} \mathrm{Y}^{2-}$. Para uma massa $\mathrm{m}_{\mathrm{EDTA} 1}$, em $\mathrm{g}$, a quantidade de matéria (número de mols) de EDTA, $\mathrm{n}_{\mathrm{EDTA}}$, é, pois, equivalente à quantidade de matéria (número de mols) do hidróxido empregado na neutralização. Assim, pelo volume de equivalência de $\mathrm{V}_{\mathrm{OH}-} / \mathrm{mL}$ consumidos do hidróxido padrão de concentração $\mathrm{C}_{\mathrm{OH}-} / \mathrm{mol} \mathrm{L}^{-1}$, tem-se:

$\mathrm{n}_{\mathrm{EDTA}}=\frac{\mathrm{V}_{\mathrm{OH}-} \mathrm{C}_{\mathrm{OH}-}}{2000}$

Multiplicando a quantidade de matéria (número de mols) de EDTA, $\mathrm{n}_{\mathrm{EDTA}}$, pela massa molar do sólido anidro, $\mathrm{W}_{\mathrm{EDTA} 1}(336,21 \mathrm{~g}$ $\mathrm{mol}^{-1}$ ), chega-se ao valor da massa do sólido anidro, $\mathrm{m}_{\mathrm{EDTA} 2}$, contido na amostra hidratada. A diferença entre as duas massas, $\mathrm{m}_{\mathrm{EDTA}}$ e $\mathrm{m}_{\mathrm{EDTA}}$, corresponde à massa de água de hidratação, a qual dividida pela massa molar da água, $\mathrm{W}_{\mathrm{H} 2 \mathrm{O}}$ fornece a quantidade de matéria (número de mols) de água, $\mathrm{n}_{\mathrm{H} 2 \mathrm{O}}$ :

$\mathrm{m}_{\mathrm{EDTA} 2}=\mathrm{n}_{\text {EDTA }} \mathrm{W}_{\text {EDTA } 1}$
$\mathrm{n}_{\mathrm{H} 2 \mathrm{O}}=\frac{\mathrm{m}_{\text {EDTA } 1 \_} \mathrm{m}_{\text {EDTA } 2}}{\mathrm{~W}_{\mathrm{H} 2 \mathrm{O}}}$

A relação entre a quantidade de matéria (número de mols) de água, $\mathrm{n}_{\mathrm{H} 2 \mathrm{O}}$, com a quantidade de matéria (número de mols) de EDTA, $\mathrm{n}_{\mathrm{EDTA}}$, indica o número de moléculas de hidratação do sólido, $\mathrm{n}_{\text {HIDRAT: }}$ :

$\mathrm{n}_{\text {HIDRAT }}=\frac{\mathrm{n}_{\mathrm{H} 2 \mathrm{O}}}{\mathrm{n}_{\mathrm{EDTA}}}$

A massa molar média do sal parcialmente hidratado, $\mathrm{W}_{\mathrm{EDTA} 2}$, resulta da seguinte Equação: 
$\mathrm{W}_{\mathrm{EDTA} 2}=\mathrm{W}_{\mathrm{EDTA} 1}+\mathrm{n}_{\mathrm{HIDRAT}} \mathrm{W}_{\mathrm{H} 2 \mathrm{O}}$

Obtendo-se o grau de hidratação do sólido de EDTA na sua forma di-sódica, $\mathrm{n}_{\text {HIDRAT }}$, e também sua respectiva massa molar média, $\mathrm{W}_{\text {EDTA2}}$, torna-se, possível a utilização deste reagente como um padrão secundário para preparo de soluções. A confiabilidade deste dado $\mathrm{W}_{\mathrm{EDTA} 2}$ reside no fato de que o ponto final detectado de forma visual ou potenciométrico é preciso, além do que o título do hidróxido padrão é obtido facilmente com 4 algarismos significatívos e incerteza de $\pm 0,1 \%$.

Facilita os cálculos o uso de um programa em linguagem QuickBASIC desenvolvido neste grupo de pesquisa, EDTAAQUA.BAS, proporcionando uma rápida análise dos dados experimentais obtidos. As saídas de dados do programa para um determinado cálculo típico são mostradas a seguir.

\section{PROGRAMA EDTAAQUA}

Calcula o grau de hidratação, massa molar média e \% de EDTA anidro Titulando com base forte

Titulo da base titulante, mol/L: 0,1896

ANÁLISE FEITA:

Massa analisada em g: 0,7044

Volume gasto do titulante, mL: 20,08

RESULTADOS:

Moléculas de água/EDTA: 1,876

Massa molar média: 370,006

Porcentagem do EDTA anidro: 90,866

Dois tipos de sólidos, A (Merck) e B (Synth), foram analisados visando a aplicação destes reagentes analíticos como padrões secundários no preparo de soluções. A Tabela 1 mostra os parâmetros analisados para as amostras $\mathrm{A}$ e $\mathrm{B}$, sendo $\mathrm{n}_{\text {HIDRAT }}$ o número de moléculas de água de hidratação do sólido, $\mathrm{W}_{\mathrm{EDTA} 2}$ a massa molar média e \%EDTA ${ }_{\text {anid }}$ a porcentagem de EDTA anidro.

Tabela 1. Parâmetros obtidos para os sólidos do tipo A e B

\begin{tabular}{lccc}
\hline AMOSTRA & $\begin{array}{c}\mathrm{n}_{\text {HIDRAT }} / \\
(\mathrm{mol})\end{array}$ & $\begin{array}{c}\mathrm{W}_{\mathrm{EDTA} /} / \\
\left(\mathrm{g} \mathrm{mol}^{-1}\right)\end{array}$ & $\begin{array}{c}\% \mathrm{EDTA}_{\text {anid }} / \\
(\%)\end{array}$ \\
\hline Sólido A (Merck) & $1,877 \pm 0,005$ & $370,02 \pm 0,09$ & $90,86 \pm 0,02$ \\
Sólido B (Synth) & $1,256 \pm 0,006$ & $358,83 \pm 0,11$ & $93,69 \pm 0,03$ \\
\hline
\end{tabular}

As medidas foram realizadas em triplicata e os dados foram analisados para um nível de confiança de $95 \%$. Analisando a Tabela 1 , verifica-se uma considerável diferença nos valores de hidratação, $\mathrm{n}_{\text {HIDRAT }}$, para as amostras dos sólidos dos tipos $\mathrm{A}$ e $\mathrm{B}$, afetando a massa molar média, $\mathrm{W}_{\text {EDTA2 }}$ que diferem do valor nominal do reagente di-hidratado, $\mathrm{Na}_{2} \mathrm{H}_{2} \mathrm{Y} .2 \mathrm{H}_{2} \mathrm{O}$. Neste caso, sem uma análise prévia, seria totalmente inviável a utilização de qualquer um destes tipos de reagentes no preparo das soluções padrão, diante dos erros relativos que poderão surgir nos valores de concentração das soluções, devido à instabilidade da composição deste reagente analítico. Por ex., no preparo de uma solução do sólido do tipo A de concentração $0,1 \mathrm{~mol} \mathrm{~L}^{-1}$, considerar esse sólido como sendo di-hidratado, $\mathrm{Na}_{2} \mathrm{H}_{2} \mathrm{Y} \cdot 2 \mathrm{H}_{2} \mathrm{O}$, levaria a um desvio no valor da concentração da ordem de $0,6 \%$, sendo que para o sólido B este desvio seria elevado para cerca de 3,7\%. Desta forma, reforça-se a importância da determinação do grau de hidratação e da massa molar média do reagente EDTA na sua forma di-sódica sempre que utilizado como padrão no preparo imediato de soluções.

Cabe acrescentar que um dos sólidos acima (sólido B) sofreu processo de recristalização, laborioso, consumindo tempo. Sua análise pelo procedimento alcalimétrico proposto revelou um valor próximo ao nominal, sendo o grau de hidratação $\mathrm{n}_{\text {HIDRAT }}=2,05$. A massa molar média calculada foi $\mathrm{W}_{\mathrm{EDTA} 2}=373,01$. Este dado corresponde a um valor $0,21 \%$ acima do valor nominal para o di-hidrato.

\section{CONCLUSÃO}

A titulação alcalimétrica proposta para o íon $\mathrm{H}_{2} \mathrm{Y}^{2-}$, em presença de íons de bário, apresenta nítido ponto final podendo ser detectado de forma visual (fenolftaleína) ou potenciométrica. Este método permite determinar o teor ativo de EDTA no sal hidratado, homogeneizado, o qual pode funcionar como um padrão secundário para preparo de suas soluções padrão. Assim, dispensa-se longo procedimento experimental para se obter o di-hidrato na sua forma ideal, isto é, $\mathrm{Na}_{2} \mathrm{H}_{2} \mathrm{Y} \cdot 2 \mathrm{H}_{2} \mathrm{O}$, sem garantia de obtê-lo com esta composição confiável.

\section{AGRADECIMENTOS}

Aos auxílios fornecidos por CNPq, CAPES e FAPESP em bolsas para pós-graduandos e recursos para o laboratório de pesquisa.

\section{REFERÊNCIAS}

1. Wheelwright, J. E.; Spedding, H. F.; Schwarzenbach, G.; J. Am. Chem. Soc. 1953, 75, 17.

2. Ringbom, A.; Complexation in Analytical Chemistry, A Guide for the Critical Selection of Analytical Methods based on Complexation Reactions, Interscience: New York, 1963.

3. Martell, E. A.; Calvin, M.; Chemistry of the Metal Chelate Compounds, Prentice-Hall: New York, 1953.

4. Chaberek, S.; Martell, E. A.; Organic Sequestring Agents, Wiley: London, 1959.

5. Flaschka, H. A.; EDTA Titrations, $2^{\text {nd }}$ ed., Pergamon Press: Londres, 1964.

6. Skoog, D. A.; West, D. M.; Holler, F. J.; Fundamentals of Analytical Chemistry; $7^{\text {th }}$ ed., Sauders College Publishing: New York, 1996.

7. Durval, C.; Anal. Chim. Acta 1957, 16, 545.

8. Fiorucci, A. R.; Saran, L. M.; Cavalheiro, E. T.; Neves, E. A.; Thermochim. Acta 2000, 356, 71.

9. Mendham, J.; Denney, R. C.; Barnes, J. D.; Thomas, K. J. M.; Vogel: Análise Química Quantitativa, $6^{\text {th }}$ ed., LTC - Livros Técnicos e Científicos: Rio de Janeiro, 2002.

10. Wendlandt, W. W.; Anal. Chem. 1960, 32, 848.

11. Oliveira, A. P.; Dissertação de Mestrado, Universidade Federal de São Carlos, Brasil, 2004.

12. Neves, E. A.; Oliveira, A.; Nóbrega, J.; The Chem. Educator 2005, 10, 5.

13. Neves, E. A; Silva, P. H. P; Oliveira, A.; Resumos do XIII Encontro Nacional de Química Analítica, Niterói, Brasil, 2005. 